\title{
Optimized Transmission of Images with OFDM over AWGN Channel Using Trigonometric Transforms
}

\author{
DewalA. Patne ${ }^{1}$, P. N. Pusdekar ${ }^{2}$ \\ ${ }^{1}$ P.G student, Amravati University, P. R. Pote (Patil) Welfare \& Education Trust"s college of Engineering \& Management, \\ Amravati, Maharashtra, India \\ ${ }^{2}$ Assistant Professor, Amravati University, P.R. Pote (Patil) Welfare \& Education Trust"s college of Engineering \& Management, \\ Amravati, Maharashtra, India
}

\begin{abstract}
A new scheme is proposed for progressive image transmission over coded Orthogonal Frequency Division Multiplexing (OFDM) system with Low Density Parity Check Coding (LDPC). Trigonometric transforms are used in this scheme for improving the performance of the OFDM systems and reducing the Peak-to-Average Power Ratio (PAPR) of OFDM signal. It improves the error resilience ability and transmission efficiency for progressive image transmission over Additive White Gaussian Noise (AWGN) channel. The Set Partitioning in Hierarchical Trees (SPIHT) algorithm is used for source coding of the images to be transmitted. In the proposed scheme the transmit data sequence of the OFDM signal after Inverse Fast Fourier Transform (IFFT) is grouped into in-phase and inquadrature components, then each component is transformed using either the Discrete Cosine Transform (DCT) or the Discrete Sine Transform (DST). The results show that adding the DST to the system improves the visual quality of reconstructed images and reduces the PAPR of OFDM signal. The major obstacle is the high peak-to-average power ratio (PAPR) in the OFDM signal causing nonlinearity at the receiving end. This paper focuses on the fact of greatly reducing the PAPR present in the OFDM signal.
\end{abstract}

Keywords: OFDM, PAPR, LDPC, SPIHT, Trigonometric Transforms

\section{Introduction}

The rapid growth of wireless communication has resulted in a demand for robust transmission of compressed images over wireless channels. Challenge is to protect the compressed image data against loss in such a way so we can maximize the received image quality. New techniques have been developed for digital transmission to fulfill the need of higher data rates in communications which can be used in both wired and wireless environments. A promising modulation technique increasingly being adopted in the telecommunication field is Orthogonal Frequency Division Multiplexing (OFDM)[1], a multicarrier Modulation technique in which a single high rate data-stream is divided into multiple low rate data-streamsand is modulated using sub-carriers which are orthogonal to each other. The carriers can be made orthogonal by suitably choosing the frequency spacing between them. OFDM offer advantages like high spectral efficiency, robustness to channel fading, immunity to impulse interferences, flexibility and easy equalization. Inspite of these benefits the major obstacle in using OFDM is that, OFDM signal exhibits very high Peak-to-Average Power Ratio (PAPR) which is the relation between the maximum powers of a sample in a given OFDM transmit symbol divided by the average power of that OFDM symbol. The PAPR occurs when in a multi-carrier system the different sub-carriers are out of phase with each other. When all the points achieve the maximum value simultaneously; this will cause the output envelope suddenly to shoot up which causes a ,peak ${ }^{\text {ee }}$ in the outp ut envelope. This peak value can be very high as compared to the average of the whole system. This ratio of the peak to average power value is termed as ,Peak-to-Average Power Ratio ${ }^{\text {ec }}$.
1.1 Previous techniques to reduce the PAPR in the OFDM system

Several techniques to reduce the PAPR in OFDM systems [2, 3] are clipping [4], companding [5, 6] Partial Transmit Sequence (PTS) [7], Selected Mapping (SLM) [8] and coding [9]. The clipping technique is the simplest one that can be used in OFDM systems, but it causes additional clipping which degrades the system performance. Signal transformation is one of the techniques to deal with high PAPR involving signal transformation prior to amplification, then an inverse transformation at the receiver prior to demodulation. The authors in [10] concluded that OFDM systems with trigonometric transforms provide higher PAPR reduction than the standard FFT based system. However, they modified the OFDM symbols before transmission using the PTS. Their results reveal that without PTS, the distribution of PAPR is the same for that conventional one, such that the reduction depends on PTS, which makes redundancy in the system. Hence it can be concluded that for OFDM signal PAPR reduction techniques are based on complexity, bandwidth expansion, spectral release \& performance characteristics.

\subsection{Why is LDPC used for coding in this scheme?}

LDPC is one of the good candidates for high speed broadband wireless applications. These are a class of linear block codes which provide a reliable transmission for coding that is very close to Shannon "s limit and also can beat Turbo codes at long block length but with relatively low decoding complexity. A (N, K) LDPC code can be represented by a very scarce parity-check matrix having $\mathrm{M}$ rows, $\mathrm{N}$ column and code rate $\mathrm{R}=\mathrm{K} / \mathrm{N}$, where $\mathrm{K}=\mathrm{N}-\mathrm{M}$. It was originally 


\section{International Journal of Science and Research (IJSR) \\ ISSN (Online): 2319-7064 \\ Index Copernicus Value (2013): 6.14 | Impact Factor (2015): 6.391}

invented by Gallager in 1963[11] and rediscovered recently by Mackay and Neal [12]. Hence the combination of the coded OFDM with LDPC (LDPC-COFDM) is used to improve the BER by adaptive bit loading and power allocation of each subcarrier.

\subsection{Image Compression Algorithm Used}

Image compression is a typical application of wavelets in digital signal processing (DSP). Multimedia data requires considerable storage capacity \& transmission bandwidth. Compression offers a means to reduce the cost of storage \& increases the speed of transmission. Image compression minimizes the size in bytes of a graphics file without degrading the quality of the received image. Embedded Zero tree Wavelet (EZW) given by Shapiro gives excellent compression performance. SPIHT is the further enhancement of EZW devised by Said and Pearlman which includes faster implementation. It is computationally very fast and among the best image compression algorithms known today.It defines parent-children relationships between the self-similar sub-bands and hence called ,Set Partitioning in Hierarchical Trees $^{\text {ee }}$. For image transmission over the OFDM system SPIHT algorithm is suggested in several research works [13, 14] because the SPIHT has a good rate-distortion performance for motionless images with comparatively low complexity and it is scalable or completely embeddable.

\section{Set Partitioning In Hierarchical Trees (SPIHT) Algorithm}

Image compression algorithm based on Discrete Wavelet Transform (DWT) is Embedded Zero tree Wavelet (EZW) [15]which gives exceptional compression performance, both in terms of statistical PSNR and subjective human perception of the reconstructed image. SPIHT is the further development of EZW algorithm. The SPIHT algorithm has been introduced by Amir Said and William Pearlman [16] in 1996 article named as „SPIHT ${ }^{e}$. One of the most important characteristics of DWT is multi-resolution decomposition. There are various wavelet based image compression algorithms which are JPEG 2000, MPEG, EZW, SPIHT, etc. JPEG and MPEG produce annoying visual degradation when operating at low bit-rates \&because they introduce errors in visually important parts of the image structure \&also introduces the blocking artifacts in the reproduced image these are less preferred.

SPIHT is one of the best algorithms in terms of the peak signal-to-noise ratio (PSNR) and execution time. This algorithm also provides excellent rate distortion performance with low encoding complexity.

SPIHT introduces three lists:

a) List of Significant Pixels (LSP),

b) List of Insignificant Pixels (LIP) and

c) List of Insignificant Sets (LIS).

First initialization is done, and then algorithm takes two stages for each level of threshold: 1. the sorting pass (in which lists are organized) and 2 . The refinement pass.
Type B (all grand-children \& further descendantsare set to zero).

SPIHT algorithm defines four types of sets, which are sets of coordinates of coefficients:

$\mathrm{O}(\mathrm{i}, \mathrm{j})$ : set of coordinates of all offspring of node $(i, j)$; children only

D $(i, j)$ : set of coordinates of all descendants of node $(i, j)$; children, grandchildren, great-grand-children, etc.

$\mathrm{H}(\mathrm{i}, \mathrm{j})$ : set of all tree roots (nodes in the highest pyramid level); parents

$\mathrm{L}(\mathrm{i}, \mathrm{j}): \mathrm{D}(\mathrm{i}, \mathrm{j})$ - O(i,j) (all descendants except the offspring); grandchildren, great-grand, etc.

To find the number of passes we use the formula $\mathrm{n}=\left[\log _{2} \mathrm{C} \max \right]$

We find initial threshold as $\mathrm{T}_{0}=2^{\mathrm{n}}$.

The following shows theoriginal \&compressedgray-scale imageusing SPIHT compression algorithm.

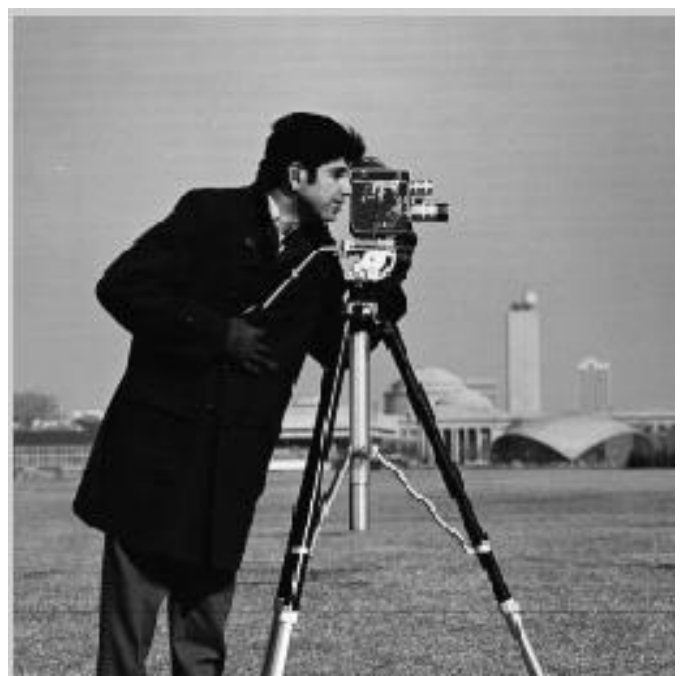

Figure 1: Original Image input at Transmitter side

After undergoing through following SPIHT encoding technique;

\section{Encoding:}

Step 1: initialization

Choose threshold $\mathrm{T}_{0}=2^{\left[\log _{2} \mathrm{c} \max \right]}$

Step 2: load the LIP with $\{(0,0),(0,1),(1,0),(1,1)\}$

Step3: load LIS with descendants i.e. $\{\mathrm{D}(0,1), \mathrm{D}$ $(1,0), D(1,1)\}$.

Step 4: LSP=Empty.

Step 5: Process LIP

If $(0,0)>\mathrm{T}_{0}$, we transmit 1 and load LSP with $(0,0)$;

If $(0,0)>0$ we transmit 0 .

Step 6: Process LIS if $\mathrm{D}(0,1), \mathrm{D}(1,0), \mathrm{D}(1,1)<\mathrm{T}_{0}$ transmit 0 .

We get a bit stream.

This bit stream can be converted back into image format after applying SPIHT encoding algorithm. The following figure 2 shows the SPIHT encoded image. 


\section{International Journal of Science and Research (IJSR) \\ ISSN (Online): 2319-7064 \\ Index Copernicus Value (2013): 6.14 | Impact Factor (2015): 6.391}

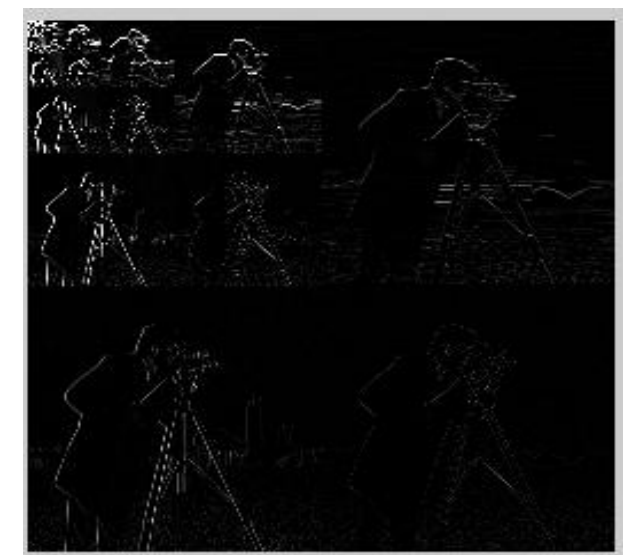

Figure 2: SPIHT applied Cameraman image

The SPIHT algorithm forms a hierarchical quad tree data structure for the wavelet transformed coefficients. The set of root node and corresponding descendants are together called as spatial orientation tree (SOT).The original but compressed image can be retrieved at the receiver end by using the decoding technique. Also the image showing error in the received image is obtained.

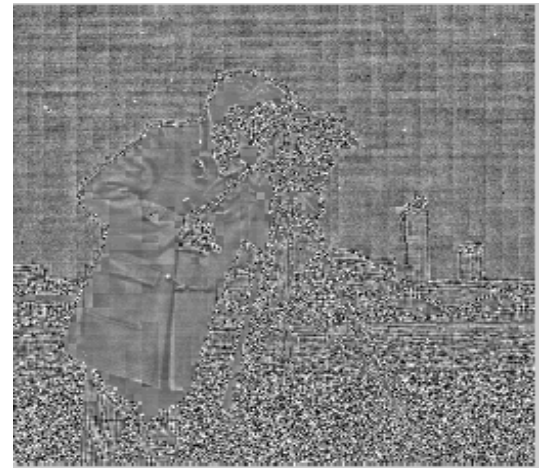

Figure 3: Error in received image

The main aim of this paper was to reduce the PAPR value of transmitted analog signal using the trigonometric transforms, so according to [17] the PAPR can be mathematically expressed as follows: $P A P R=\frac{\max \left|x_{g}(t)\right|^{2}}{E\left[\left|x_{g}(t)\right|^{2}\right]}$

Where E[.] is the average power. Generally, the PAPR is considered for a single OFDM symbol, which has a time duration $T$.Various different gray-scale and true color (RGB) images can be used as an input image with 8 bits per pixel; these images can be obtained from MATLAB toolbox with a resolution of 256 x 256. The fidelity of it was measured by the Peak Signal-To-Noise Ratio (PSNR). This PSNR is mathematically defined as follows:

$$
P S N R=10 \log _{10}\left(\frac{\text { Peak }^{2}}{M S E}\right)
$$

Where, MSE is the mean squared error between the original and the reconstructed image, and Peak is the maximum possible magnitude for a pixel inside the image. The peak value is 255 for an 8 bits/pixel of original image.

\section{Algorithm for LDPC COFDM system model flow with Trigonometric Transforms}

Step 1:Get the input gray-scale image of256 x 256 dimensions for transmission. If it is a true color image then getting it converted from true color (RGB)to gray-scale.
Step 2: Applying SPIHT encoding algorithm which divides image stream into several layers \& getting it converted to a binary format.

Step 3: These information bits are then encoded with LDPC encoder.

Step 4: Since OFDM is utilizing,$N^{\mathrm{ee}}$ number of subcarriers so baseband data is converted to parallel data. These ${ }_{2} \mathrm{~N}^{\mathrm{ee}}$ subcarriers are chosen to be orthogonal.

Step 5: The transmitted data is modulated by Binary Phase Shift Keying (BPSK).

Step 6: The modulated data undergo Inverse Fast Fourier Transform (IFFT) \& gets split into in-phase component, inquad component.

Step 7: DST is applied to both components which reduces the PAPR at the receiver end.

Step 8: At the receiver end the decoding is carried out \&the original compressed image is retrieved, also an image showing error is obtained.

Step 9: At last the PSNR value\&the PAPR value of the received image is also observed.

The following table shows different images with their PSNR \& PAPR values in $\mathrm{dB}$ both at the transmitter $\left(\mathrm{T}^{\mathrm{x}}\right)$ side \& receiver $\left(\mathrm{R}^{\mathrm{X}}\right)$ side resulted on running the MATLAB code.

Table 1: Comparing PSNR \& PAPR values at $T^{\mathrm{x}} \& \mathrm{R}^{\mathrm{x}}$ sides

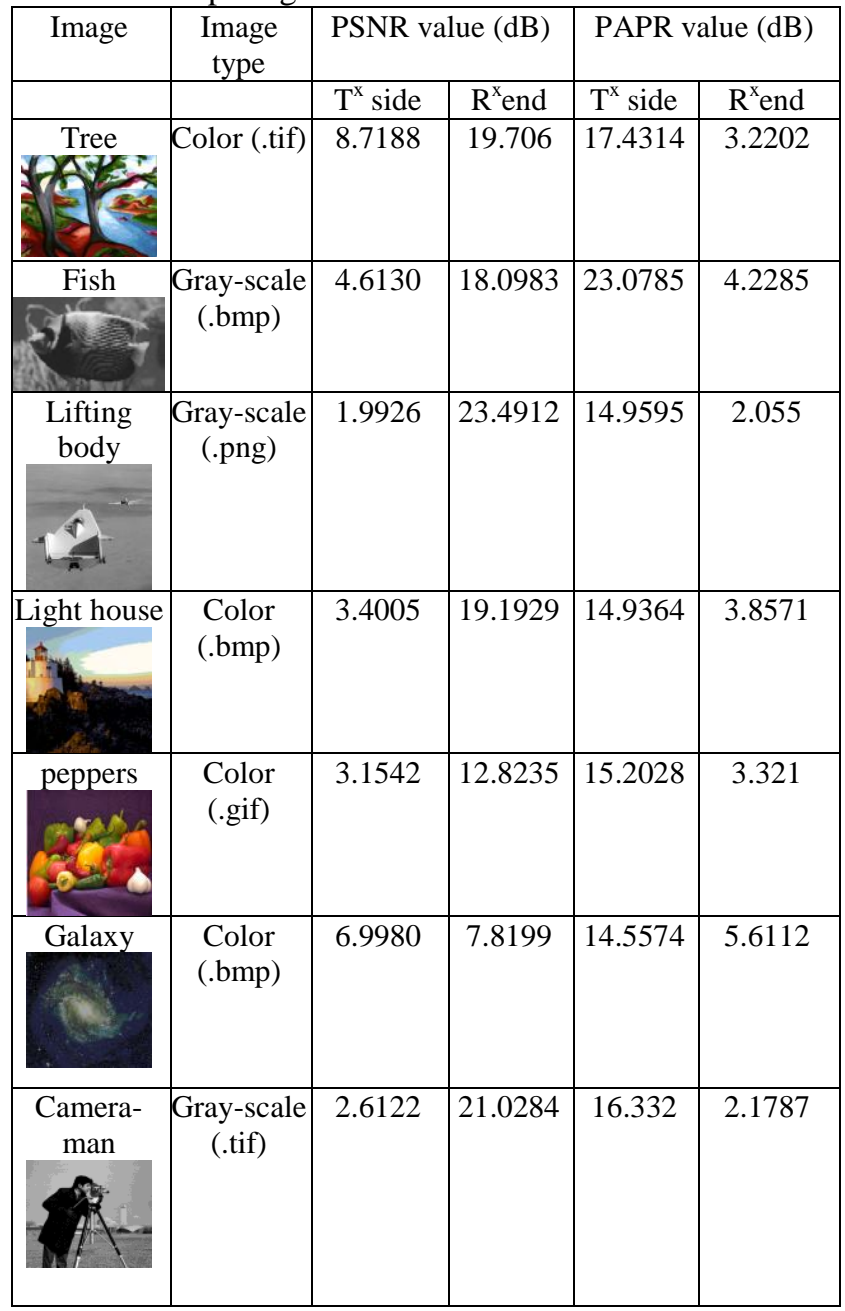




\section{International Journal of Science and Research (IJSR) \\ ISSN (Online): 2319-7064 \\ Index Copernicus Value (2013): 6.14 | Impact Factor (2015): 6.391}

\section{Conclusion}

In this paper, an efficient LDPC coded OFDM system with trigonometric transforms (DCT/DST)which supports image transmission using SPIHT compression technique is advised. The effectiveness of the proposed system can be observed from the table showing comparison of PSNR \& PAPR values at transmitter and receiver sides obtained after running the MATLAB code. The reduced PAPR value obtained at the receiver endproves that, the major obstacle in the coded OFDM signalis greatly reduced when it is designed with the Discrete Sine Transform (DST)for the transmitted signal.

\section{References}

[1] R. W Chang, “Orthogonal Frequency Division Multiplexing”, U.S Patent 3388455, Jan 6, 1970, Filed Nov.4. 1966.

[2] T. Jiang and Y. Wu, "An Overview: Peak-to-Average Power Ratio Reduction Techniques for OFDM Signals", IEEE Transactions on Broadcasting, Vol. 54, No. 2, pp. 257-268, Jun. 2008.

[3] F.S. AI-Kamali, M. I. Dessouky, B.M. Sallam, F. Shawki and F. E. Abd EI-Samie, "Transceiver Scheme For Single-Carrier Frequency Division Multiple Access Implementing the Wavelet Transform and Peak-ToAverage-Power Ratio Reduction Methods" IET Communications, Vol. 4, No. 1, pp. 69-79, 2010.

[4] J. Kim and Y. Shin, "An Effective Clipped Companding Scheme for PAPR Reduction of OFDM Signals", Proceedings of the IEEE ICC'08, pp.668-672, 2008.

[5] T. Jiang, W. Yao, P. Guo, Y. Song and D. Qu, "Two Novel Nonlinear Companding Schemes With Iterative Receiver to Reduce PAPR in Multi-Carrier Modulation Systems", IEEE Transactions on Broadcasting, Vol. 51, No. 2, pp. 268 - 273, Jun. 2006.

[6] F. S. AI-Kamali, M. I. Dessouky, B. M. Sallam, F. Shawki and F. E. Abd EI-Samie, "Performance Enhancement of SC-FDMA Systems Using a Companding Technique", Ann. Telecommun. , Vol. 65, No. 5-6, pp. 293-300, 2010.

[7] L. Guan, T. Jiang, D. Qu and Y. Zhou, "Joint Channel Estimation and PTS to Reduce Peak-to-Average-Power Ratio in OFDM Systems without Side Information", IEEE Signal Processing Letters, Vol. 17, No. 10, pp. 883-886, October 2010.

[8] E. S. Hassan, S. E. EI-Khamy, M. I. Dessouky, S. A. EIDolil and F. E. Abd EI-Samie, "A Simple Selective Mapping Algorithm For The Peak-To-Average Power Ratio In Space Time Block Coded MIMO-OFDM Systems" Proceedings of the International Conference on High Performance Computing, Networking and Communication Systems (HPCNCS-08),2008.

[9] T. Jiang and G. Zhu, "Complement Block Coding for Reduction in Peak-to-Average Power Ratio of OFDM Signals," IEEE Communications Magazine, vol. 43, no.9, pp. S17 - S22, Sept. 2005.

[10]A. Rushdi and J. Tuqan, "Peak reduction in Trigonometric Based OFDM System", Proceedings in ACSSC.2007, pp. 1747 - 1751, 2007.

[11]R G. Gallagher, "Low Density Parity Check Codes", IRE Trans. Inform. Theory, Vol. IT-8, pp. 21-28, Jan. 1962.

[12] D. J. C. MacKay, "Good Error-
Correcting Codes Based on Very Sparse Matrices", IEEE Trans. Inform. Theory, Vo1.45, pp. 399-43 1, Mar. 1999.

[12]Y. Sun, X. Wang and Liu, K.J.R, "A Joint Channel Estimation and Unequal Error Protection Scheme for Image Transmission in Wireless OFDM Systems", Multimedia Signal Processing, IEEE, pp. 380 - 383, 2002.

[13] S. Wang, J. Dai, C. Hou and X. Liu "Progressive Image Transmission over Wavelet Packet Based OFDM", Proceedings in Electrical and Computer Engineering 2006 Canadian Conference, pp. 950 - 953, 2006.

[14] J.M. Shapiro, "Embedded image coding using the zero trees of wavelet coefficients", IEEE Transactions on Image Processing, Vol. 41, No. 12, pp. 3445-3462, December 1993.

[15] Said Amir and Pearlman William A., "A New Fast and Efficient Image Codec Based on Set Partitioning in Hierarchical Trees" ,IEEE Transactions On Circuits and Systems For VideoTechnology, Vol.6, Pg. No. 243-250, June 1996.

[16]H. Schulze and C. Luders, Theory and Application of OFDM and CDMA Wideband Wireless Communication John Wiley, 2005. 